Article

\title{
Elementary Bounds on the Ruin Capital in a Diffusion Model of Risk
}

\section{Vsevolod K. Malinovskii}

Central Economics and Mathematics Institute (CEMI) of Russian Academy of Science, Nakhimovskiy prosp., 47, Moscow 117418, Russia; E-Mail: malinov@ orc.ru or admin@actlab.ru; Tel.:+8-985-143-3783

Received: 29 March 2014; in revised form: 19 June 2014 / Accepted: 30 June 2014 /

Published: 8 July 2014

\begin{abstract}
In a diffusion model of risk, we focus on the initial capital needed to make the probability of ruin within finite time equal to a prescribed value. It is defined as a solution of a nonlinear equation. The endeavor to write down and to investigate analytically this solution as a function of the premium rate seems not technically feasible. Instead, we obtain informative bounds for this capital in terms of elementary functions.
\end{abstract}

Keywords: level premium rate; level initial capital; diffusion model of risk

\section{Introduction and Rationale}

In this paper, we focus on the solutions (w.r.t. $u$ and $c$ ) of the nonlinear equation

$$
\boldsymbol{\psi}_{t}(u, c \mid \vartheta)=\alpha
$$

where $\alpha>0$ is a sufficiently small number and $\boldsymbol{\psi}_{t}(u, c \mid \vartheta)$ is the probability of ruin within finite time $t>0$ in the standard diffusion risk model, i.e., when the annual risk reserve at time $s \geqslant 0$ is defined as

$$
R_{s}=u+c s-V_{s}, \quad V_{s}=\vartheta s+\sigma(\vartheta) \mathbf{W}_{s}
$$

Here $u>0$ is the initial risk reserve and $c>0$ is the premium rate. By $\mathrm{W}_{s}, s \geqslant 0$, we denote a standard Brownian motion. The aggregate claims payout process $V_{s}, s \geqslant 0$, is a diffusion with continuous time, starting at zero. Its drift coefficient is $\vartheta>0$ and diffusion coefficient is $\sigma(\vartheta)>0$. The variance is $\mathrm{D} V_{s}=\sigma^{2}(\vartheta) s$. 
Bearing in mind the well known result for diffusion with linear drift (see, e.g., Equation 1.1.4 in Part II, Chapter 2 of [1]), we have in this model

$$
\begin{aligned}
& \boldsymbol{\psi}_{t}(u, c \mid \vartheta)= \mathrm{P}\left\{\inf _{0<s \leqslant t}\left[u+(c-\vartheta) s-\sigma(\vartheta) \mathrm{W}_{s}\right]<0\right\} \\
&=1-\Phi_{(0,1)}\left(\frac{u}{\sigma(\vartheta) \sqrt{t}}-\frac{(\vartheta-c) \sqrt{t}}{\sigma(\vartheta)}\right)+\exp \left\{2 \frac{u(\vartheta-c)}{\sigma^{2}(\vartheta)}\right\} \\
& \quad \times \Phi_{(0,1)}\left(-\frac{u}{\sigma(\vartheta) \sqrt{t}}-\frac{(\vartheta-c) \sqrt{t}}{\sigma(\vartheta)}\right)
\end{aligned}
$$

Here $\Phi_{(0,1)}(x)$ and $\varphi_{(0,1)}(x)$ are the standard normal c.d.f. and p.d.f. respectively.

The solution of Equation (1) with respect to $u$ is called the $\alpha$-level initial capital, or ruin capital. We denote it by $u_{\alpha, t}(c \mid \vartheta)$. The solution with respect to $c$ is called the $\alpha$-level premium rate. We denote it by $c_{\alpha, t}(u \mid \vartheta)$. Since (see Equation (3)) $\boldsymbol{\psi}_{t}(0, c \mid \vartheta) \equiv 1$ for any $t>0$, and since $\boldsymbol{\psi}_{t}(u, c \mid \vartheta)$ monotone decreases, as $u>0(c>0)$ increases, the solution $u_{\alpha, t}(c \mid \vartheta)$ exists for all $0<\alpha<1$, but only small $\alpha$ are of interest. This solution is bounded from above by $u_{\alpha, t}(0 \mid \vartheta)>0$ and monotone decreases to zero, as $c$ increases.

It is easy to see that for any $c>0$

$$
c_{\alpha, t}\left(u_{\alpha, t}(c \mid \vartheta) \mid \vartheta\right)=c
$$

and for any $u>0$

$$
u_{\alpha, t}\left(c_{\alpha, t}(u \mid \vartheta) \mid \vartheta\right)=u
$$

The inverse functions for both $u_{\alpha, t}(c \mid \vartheta)$ and $c_{\alpha, t}(u \mid \vartheta)$ obviously exist ${ }^{1}$. From Equations (4) and (5), we have $u_{\alpha, t}(c \mid \vartheta)=c_{\alpha, t}^{-1}(c \mid \vartheta)$ for any $c>0$ and $c_{\alpha, t}(u \mid \vartheta)=u_{\alpha, t}^{-1}(u \mid \vartheta)$ for any $u>0$. So, enough is to focus on the ruin capital $u_{\alpha, t}(c \mid \vartheta)$.

The idea to look at the dependence of the initial capital $u$ on the premium rate $c$, or vice versa, holding the probability of ruin $\psi_{t}(u, c \mid \vartheta)$ at a prescribed level $\alpha$, i.e., to use this probability merely as a binder, agrees with insurance regulation. In a nutshell, each insurance year the premium may be set within sensible limits, while the corresponding initial capital must be so large that the probability of ruin remains at a prescribed level.

Developing a sensible control over the years ${ }^{2}$, insurance managers are interested not in the probability of ruin as itself, but in a good balance between the control leverages, such as the initial capital and premium rate, which makes the solvency controllable. Typically, the expressions for the probability of ruin within finite time like Equation (3) are either absent or intractable, and the decision makers perform their calculations numerically ${ }^{3}$.

Even when an explicit formula like (3) exists, to find $u_{\alpha, t}(c \mid \vartheta)$ in an explicit form, using elementary functions, is a tremendous endeavor. This can not be done even in our diffusion model (2). However,

1 Being the inverse function, $c_{\alpha, t}(u \mid \vartheta)=u_{\alpha, t}^{-1}(u \mid \vartheta)$ is defined accordingly for $0<u<u_{\alpha, t}(0 \mid \vartheta)$. For completeness, it is set zero for $u>u_{\alpha, t}(0 \mid \vartheta)$.

2 In [2-4], the framework of this paper was embedded in the multi-year controlled diffusion models of risk and the controls were built annually, depending on the past years financial results.

3 This paper deals with analytical methods rather than numerical evaluation. There is no need to say about the difference between analytical and numerical analysis. 
in order to develop a sensible control, we need to scrutinize the behavior of $u_{\alpha, t}(c \mid \vartheta)$ for all possible values of $c>0$. To this end, we propose in this paper informative and elementary upper and lower bounds. These bounds are compound and give valuable information about $u_{\alpha, t}(c \mid \vartheta)$ in the both cases of profitable $(c>\vartheta)$ and unprofitable $(0<c<\vartheta)$ risk reserve process. The fundamental observation underlying the proof in the former case is the convexity (concavity downward) of $u_{\alpha, t}(c \mid \vartheta)$, as a function of $c$.

In [5], we have considered the similar problem in the classical Lundberg model. The classical Lundberg model yields the second exceptional case where the analytical expression for the probability of ruin within finite time is known. Unlike in [5], the bounds in this paper are given for each $t$ rather than asymptotically, as $t \rightarrow \infty$.

The rest of the paper is arranged as follows.

In Section 2, we formulate some background results obtained previously.

In Section 3, we formulate the main results. The fundamental remark about convexity of $u_{\alpha, t}(c \mid \vartheta)$ as a function of $c$, as $c>\vartheta$, is proved by showing that $\frac{\partial^{2}}{\partial c^{2}} u_{\alpha, t}(c \mid \vartheta)$ is positive. To find this second derivative, we apply the implicit function theorem. The other technical tool are the inequalities for the Mill's ratio proved in [6].

In Section 4, we formulate some auxiliary results.

\section{Some Background Results}

For completeness, we present a number of related results obtained in [2-4]. Because of lack of space we do not give details of their proof.

\subsection{Some Equalities}

We put $\kappa_{\gamma}=\Phi_{(0,1)}^{-1}(1-\gamma)$ for $(1-\gamma)$-quantile $(0<\gamma<1)$. If $\gamma$ increases from 0 to $1 / 2$, i.e., $1-\gamma$ decreases from 1 to $1 / 2$, the quantile $k_{\gamma}$ monotone decreases from $+\infty$ to zero. By symmetry, we have $k_{1-\gamma}=-k_{\gamma}$ and $k_{1 / 2}=0$. For $u_{\alpha, t}(\vartheta \mid \vartheta)$, we have the following representation ${ }^{4}$.

Theorem 1 (Theorem 2.1 in [2]) In the diffusion model (2), the solution with respect to u of Equation (1), as $c=\vartheta$, is

$$
u_{\alpha, t}(\vartheta \mid \vartheta)=\sigma(\vartheta) \sqrt{t} \kappa_{\alpha / 2}
$$

For $u_{\alpha, t}(c \mid \vartheta)$ and $c_{\alpha, t}(u \mid \vartheta)$, we have the following representations.

Theorem 2 (Theorem 4.4 in [3]) In the diffusion model (2), the solution with respect to u of Equation (1) is

$$
u_{\alpha, t}(c \mid \vartheta)= \begin{cases}\sigma(\vartheta) \sqrt{t}\left[\frac{(\vartheta-c) \sqrt{t}}{\sigma(\vartheta)}+\mathrm{U}_{\alpha}\left(\frac{(\vartheta-c) \sqrt{t}}{\sigma(\vartheta)}\right)\right], & c \leqslant \vartheta \\ \sigma(\vartheta) \sqrt{t} \mathrm{U}_{\alpha}\left(\frac{(\vartheta-c) \sqrt{t}}{\sigma(\vartheta)}\right), & c>\vartheta\end{cases}
$$

4 In what follows, we will make extensive use of both entries, $u_{\alpha, t}(\vartheta \mid \vartheta)$ and $\sigma(\vartheta) \sqrt{t} \kappa_{\alpha / 2}$. It will not be difficult to convert into each other the expressions using either of these two forms. 
where the function $\mathrm{U}_{\alpha}(x)$ for $x<0$ satisfies the equation

$$
1-\Phi_{(0,1)}\left(\mathrm{U}_{\alpha}(x)-x\right)+\exp \left\{2 x \mathrm{U}_{\alpha}(x)\right\} \Phi_{(0,1)}\left(-\mathrm{U}_{\alpha}(x)-x\right)=\alpha
$$

for $x \geqslant 0$ satisfies the equation

$$
1-\Phi_{(0,1)}\left(\mathrm{U}_{\alpha}(x)\right)+\exp \left\{2 x\left[x+\mathrm{U}_{\alpha}(x)\right]\right\} \Phi_{(0,1)}\left(-2 x-\mathrm{U}_{\alpha}(x)\right)=\alpha
$$

is continuous, positive for all $x$, monotone increasing, as $x$ increases from $-\infty$ to 0 , and monotone decreasing, as $x$ increases from 0 to $+\infty$, and such that ${ }^{5}$

$$
\lim _{x \rightarrow-\infty} \mathrm{U}_{\alpha}(x)=0, \quad \mathrm{U}_{\alpha}(0)=\kappa_{\alpha / 2}, \quad \lim _{x \rightarrow+\infty} \mathrm{U}_{\alpha}(x)=\kappa_{\alpha}
$$

Corollary 1 (Theorem 2.1 in [4]) In the diffusion model (2), the solution with respect to c of Equation (1) is

$$
c_{\alpha, t}(u \mid \vartheta)= \begin{cases}\vartheta-\frac{\sigma(\vartheta)}{\sqrt{t}}\left(\frac{u-u_{\alpha, t}(\vartheta \mid \vartheta)}{\sigma(\vartheta) \sqrt{t}}+\mathrm{G}_{\alpha}\left(\frac{u}{\sigma(\vartheta) \sqrt{t}}\right)\right), & u \geqslant u_{\alpha, t}(\vartheta \mid \vartheta) \\ \vartheta-\frac{\sigma(\vartheta)}{\sqrt{t}}\left(\frac{\sigma(\vartheta) \sqrt{t}}{u} \mathrm{G}_{\alpha}\left(\frac{u}{\sigma(\vartheta) \sqrt{t}}\right)\right), & u<u_{\alpha, t}(\vartheta \mid \vartheta)\end{cases}
$$

where $u_{\alpha, t}(\vartheta \mid \vartheta)=\sigma(\vartheta) \sqrt{t} \kappa_{\alpha / 2}$ and the function $\mathrm{G}_{\alpha}(x), x>0$, is continuous, monotone increasing, as $x$ increases, and such that ${ }^{6}$

$$
\lim _{x \rightarrow 0} \mathrm{G}_{\alpha}(x)=\frac{\ln \alpha}{2}, \quad \mathrm{G}_{\alpha}\left(\kappa_{\alpha / 2}\right)=0, \quad \lim _{x \rightarrow+\infty} \mathrm{G}_{\alpha}(x)=\kappa_{\alpha / 2}-\kappa_{\alpha}
$$

For $x \geqslant \kappa_{\alpha / 2}$, being positive, $\mathrm{G}_{\alpha}(x)$ is convex and satisfies the equation

$$
1-\Phi_{(0,1)}\left(\kappa_{\alpha / 2}-\mathrm{G}_{\alpha}(x)\right)+\exp \left\{2 x\left[x-\kappa_{\alpha / 2}+\mathrm{G}_{\alpha}(x)\right]\right\} \Phi_{(0,1)}\left(-2 x+\kappa_{\alpha / 2}-\mathrm{G}_{\alpha}(x)\right)=\alpha
$$

For $0<x<\kappa_{\alpha / 2}$, being negative, $\mathrm{G}_{\alpha}(x)$ is concave and satisfies the equation

$$
1-\Phi_{(0,1)}\left(x-\mathrm{G}_{\alpha}(x) / x\right)+\exp \left\{2 \mathrm{G}_{\alpha}(x)\right\} \Phi_{(0,1)}\left(-x-\mathrm{G}_{\alpha}(x) / x\right)=\alpha
$$

Moreover, for $0<x<\kappa_{\alpha / 2}$ we have

$$
\mathrm{G}_{\alpha}(x) \leqslant\left(1-\frac{x}{\kappa_{\alpha / 2}}\right) \frac{\ln \alpha}{2}
$$

Since the functions $\mathrm{U}_{\alpha}(\cdot)$ and $\mathrm{G}_{\alpha}(\cdot)$ can not be written explicitly, Theorem 2 and Corollary 1 do not give the explicit solutions of Equation (1).

5 Since $0<\alpha<\frac{1}{2}$, one has $0<\kappa_{\alpha}<\kappa_{\alpha / 2}<1$.

6 Recall that since $0<\alpha<\frac{1}{2}$, we have $0<\kappa_{\alpha}<\kappa_{\alpha / 2}<1$. 


\subsection{Some Inequalities}

The following upper and lower bounds on $u_{\alpha, t}(c \mid \vartheta)$ follow from Theorem 2 . They are illustrated in Figure 1.

Figure 1. The function $u_{\alpha, t}(c \mid \vartheta)$ of the variable $c>0$ (X-axis), as $\vartheta=1, \alpha=0.1$, $t=200$, and the bounds of Theorem 3. Horizontal line: $u_{\alpha, t}(\vartheta \mid \vartheta)=\sigma(\vartheta) \sqrt{t} \kappa_{\alpha / 2}$.

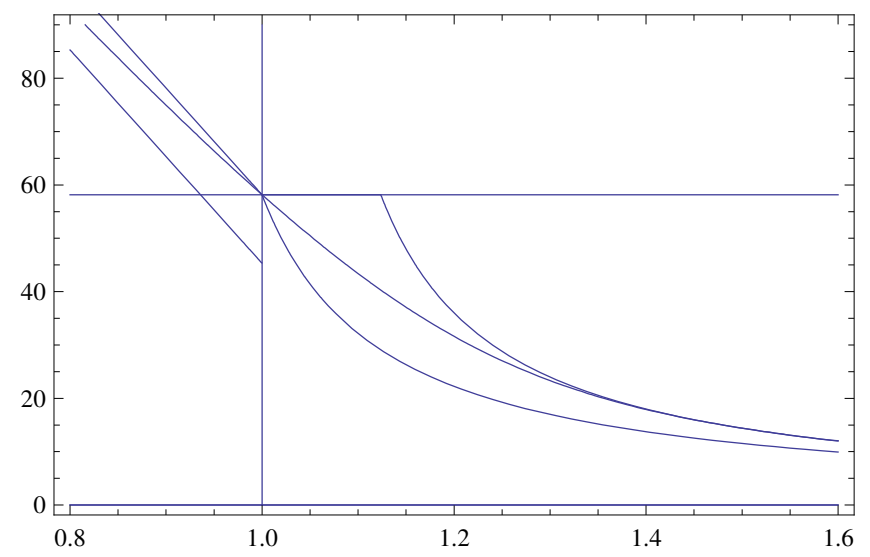

Theorem 3 In the diffusion model (2), the solution with respect to $u$ of Equation (1) is such that for $0<c<\vartheta$

$$
\sigma(\vartheta) \sqrt{t}\left(\frac{(\vartheta-c) \sqrt{t}}{\sigma(\vartheta)}+\kappa_{\alpha}\right) \leqslant u_{\alpha, t}(c \mid \vartheta) \leqslant \sigma(\vartheta) \sqrt{t}\left(\frac{(\vartheta-c) \sqrt{t}}{\sigma(\vartheta)}+\kappa_{\alpha / 2}\right)
$$

and for $c>\vartheta$

$$
-\frac{\sigma^{2}(\vartheta) \ln \alpha}{2}\left(c-\vartheta-\frac{\sigma(\vartheta) \ln \alpha}{2 \sqrt{t} \kappa_{\alpha / 2}}\right)^{-1} \leqslant u_{\alpha, t}(c \mid \vartheta) \leqslant \min \left\{u_{\alpha, t}(\vartheta \mid \vartheta),-\frac{\sigma^{2}(\vartheta) \ln \alpha}{2}(c-\vartheta)^{-1}\right\}
$$

It is noteworthy (see Figure 1) that the upper bound for $c>\vartheta$ in Theorem 3 is compound: it is horizontal line until $u_{\alpha, t}(\vartheta \mid \vartheta)<-\frac{1}{2} \sigma^{2}(\vartheta) \ln \alpha(c-\vartheta)^{-1}$ and hyperbola otherwise. This hyperbola is the solution of the equation $\boldsymbol{\psi}_{\infty}(u, c \mid \vartheta)=\alpha$.

The following upper and lower bounds on $c_{\alpha, t}(u \mid \vartheta)$ follow from Corollary 1.

Corollary 2 (Corollary 2.1 in [4]) In the diffusion model (2), the solution with respect to c of Equation (1) is such that for $0<u \leqslant u_{\alpha, t}(\vartheta \mid \vartheta)$

$$
\vartheta-\frac{\sigma(\vartheta)}{\sqrt{t}}\left(\frac{\sigma(\vartheta) \sqrt{t}}{u}\left(1-\frac{u}{u_{\alpha, t}(\vartheta \mid \vartheta)}\right) \frac{\ln \alpha}{2}\right) \leqslant c_{\alpha, t}(u \mid \vartheta) \leqslant \vartheta-\frac{\sigma(\vartheta)}{\sqrt{t}}\left(\frac{\sigma(\vartheta) \sqrt{t}}{u} \frac{\ln \alpha}{2}\right)
$$

and for $u>u_{\alpha, t}(\vartheta \mid \vartheta)$

$$
\vartheta-\frac{\sigma(\vartheta)}{\sqrt{t}}\left(\frac{u-u_{\alpha, t}(\vartheta \mid \vartheta)}{\sigma(\vartheta) \sqrt{t}}+\kappa_{\alpha / 2}-\kappa_{\alpha}\right) \leqslant c_{\alpha, t}(u \mid \vartheta) \leqslant \vartheta-\frac{\sigma(\vartheta)}{\sqrt{t}}\left(\frac{u-u_{\alpha, t}(\vartheta \mid \vartheta)}{\sigma(\vartheta) \sqrt{t}}\right)
$$




\section{The Main Results}

In this section, our goal is to improve the upper bounds in Theorem 3, as $c>\vartheta$, and in Corollary 2, as $0<u \leqslant u_{\alpha, t}(\vartheta \mid \vartheta)$. This improvement makes these rather rough upper bounds much more accurate and informative.

Our plan regarding Theorem 3 is as follows. First, for $c>\vartheta$, we prove the convexity (concavity downward) of $u_{\alpha, t}(c \mid \vartheta)$, as a function of $c$. Then we enhance Theorem 3, as illustrated in Figures 2 and 3. We draw the tangent line to hyperbola $-\frac{1}{2} \sigma^{2}(\vartheta) \ln \alpha(c-\vartheta)^{-1}$ which is an upper bound for the ruin capital for $c>\vartheta$. Since $u_{\alpha, t}(c \mid \vartheta)$ is a convex function of $c>\vartheta$, this tangent line is the required upper bound on $u_{\alpha, t}(\vartheta \mid \vartheta)$ to the left of the point of tangency.

Figure 2. Graphs shown in Figure 1, with the graph of sloping straight line $t \kappa_{\alpha / 2}^{2}(c-$ $\vartheta) /(2 \ln \alpha)+\sigma(\vartheta) \sqrt{t} \kappa_{\alpha / 2}$ starting from the point with abscissa $\vartheta$ and ordinate $u_{\alpha, t}(\vartheta \mid \vartheta)=$ $\sigma(\vartheta) \sqrt{t} \kappa_{\alpha / 2}$, and tangent to the hyperbola $\left(-\sigma^{2}(\vartheta) \ln \alpha\right) /(2(c-\vartheta))$ at (right vertical line) $c=\vartheta-\sigma(\vartheta) \ln \alpha /\left(\sqrt{t} \kappa_{\alpha / 2}\right)$.

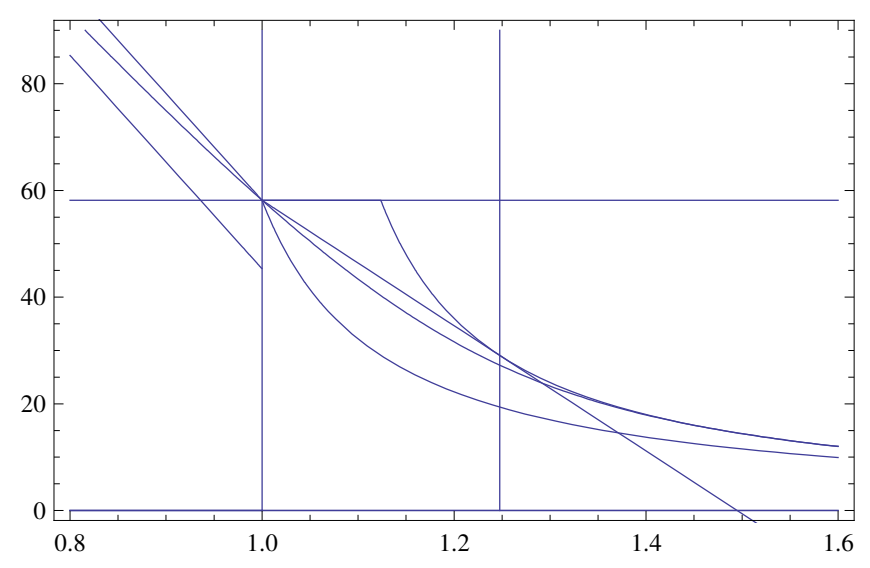

We formulate the following Theorem 4 which yields the improved upper bounds for $u_{\alpha, t}(c \mid \vartheta)$, as $c>\vartheta$. It is illustrated in Figure 3. The proof of Theorem 4 is illustrated in Figure 2 and will be presented at the end of this section.

Figure 3. The function $u_{\alpha, t}(c \mid \vartheta)$ of the variable $c>0$ (X-axis), as $\vartheta=1, \alpha=0.1$, $t=200$, and the bounds of Theorem 3 improved in Theorem 4 .

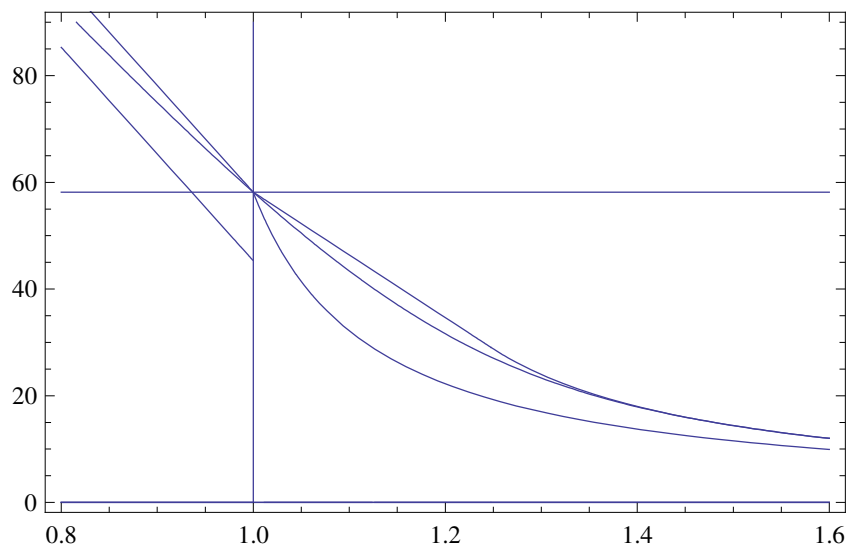


Theorem 4 In the diffusion model (2), for $c>\vartheta$ we have

$$
u_{\alpha, t}(c \mid \vartheta) \leqslant \begin{cases}\frac{t \kappa_{\alpha / 2}^{2}}{2 \ln \alpha}(c-\vartheta)+\sigma(\vartheta) \sqrt{t} \kappa_{\alpha / 2}, & \vartheta<c \leqslant \vartheta-\frac{\sigma(\vartheta) \ln \alpha}{\sqrt{t} \kappa_{\alpha / 2}} \\ -\frac{\sigma^{2}(\vartheta) \ln \alpha}{2}(c-\vartheta)^{-1}, & c>\vartheta-\frac{\sigma(\vartheta) \ln \alpha}{\sqrt{t} \kappa_{\alpha / 2}}\end{cases}
$$

The following result yields the improved upper bounds for $c_{\alpha, t}(u \mid \vartheta)$, as $0<u<u_{\alpha, t}(\vartheta \mid \vartheta)$.

Corollary 3 In the diffusion model (2), for $0<u \leqslant u_{\alpha, t}(\vartheta \mid \vartheta)$ we have

$$
c_{\alpha, t}(u \mid \vartheta) \leqslant \begin{cases}\vartheta-\frac{\sigma^{2}(\vartheta)}{u} \frac{\ln \alpha}{2}, & 0<u \leqslant \frac{u_{\alpha, t}(\vartheta \mid \vartheta)}{2} \\ \frac{2 \sigma^{2}(\vartheta) \ln \alpha}{u_{\alpha, t}^{2}(\vartheta \mid \vartheta)}\left(u-u_{\alpha, t}(\vartheta \mid \vartheta)\right)+\vartheta, & \frac{u_{\alpha, t}(\vartheta \mid \vartheta)}{2}<u \leqslant u_{\alpha, t}(\vartheta \mid \vartheta)\end{cases}
$$

The following result is fundamental.

Theorem 5 For $c>\vartheta$, the function $u_{\alpha, t}(c \mid \vartheta)$ of the variable $c$ is convex.

Proof of Theorem 5 It suffices to show that $\frac{\partial^{2}}{\partial c^{2}} u_{\alpha, t}(c \mid \vartheta)>0$. Bearing in mind Equation (1), apply Theorem 7 . We have

$$
\frac{\partial}{\partial c} u_{\alpha, t}(c \mid \vartheta)=-\frac{\left.\boldsymbol{\psi}_{t}^{(0,1)}(u, c \mid \vartheta)\right|_{u=u_{\alpha, t}(c \mid \vartheta)}}{\left.\boldsymbol{\psi}_{t}^{(1,0)}(u, c \mid \vartheta)\right|_{u=u_{\alpha, t}(c \mid \vartheta)}}
$$

and ${ }^{7}$

$$
\begin{aligned}
& \frac{\partial^{2}}{\partial c^{2}} u_{\alpha, t}(c \mid \vartheta)=-\frac{1}{\left(\left.\boldsymbol{\psi}_{t}^{(1,0)}(u, c \mid \vartheta)\right|_{u=u_{\alpha, t}(c \mid \vartheta)}\right)^{3}} \\
& \quad \times\left\{\left.\boldsymbol{\psi}_{t}^{(0,2)}(u, c \mid \vartheta)\right|_{u=u_{\alpha, t}(c \mid \vartheta)}\left(\left.\boldsymbol{\psi}_{t}^{(1,0)}(u, c \mid \vartheta)\right|_{u=u_{\alpha, t}(c \mid \vartheta)}\right)^{2}\right. \\
& \quad-\left.\left.\left.2 \boldsymbol{\psi}_{t}^{(1,1)}(u, c \mid \vartheta)\right|_{u=u_{\alpha, t}(c \mid \vartheta)} \boldsymbol{\psi}_{t}^{(1,0)}(u, c \mid \vartheta)\right|_{u=u_{\alpha, t}(c \mid \vartheta)} \boldsymbol{\psi}_{t}^{(0,1)}(u, c \mid \vartheta)\right|_{u=u_{\alpha, t}(c \mid \vartheta)} \\
& \left.\quad+\left.\boldsymbol{\psi}_{t}^{(2,0)}(u, c \mid \vartheta)\right|_{u=u_{\alpha, t}(c \mid \vartheta)}\left(\left.\boldsymbol{\psi}_{t}^{(0,1)}(u, c \mid \vartheta)\right|_{u=u_{\alpha, t}(c \mid \vartheta)}\right)^{2}\right\}
\end{aligned}
$$

Bearing in mind Equation (3), introduce ${ }^{8}$

$$
F(x, y)=1-\Phi_{(0,1)}(x+y)+\exp \{-2 x y\} \Phi_{(0,1)}(-x+y)
$$

We have

$$
\begin{aligned}
\boldsymbol{\psi}_{t}^{(1,0)}(u, c \mid \vartheta) & =\left.\frac{1}{\sigma(\vartheta) \sqrt{t}} F^{(1,0)}(x, y)\right|_{\substack{x=\frac{u}{\sigma(\vartheta) \sqrt{t}} \\
y=-\frac{\vartheta-c) \sqrt{t}}{\sigma(\vartheta)}}} \\
\boldsymbol{\psi}_{t}^{(0,1)}(u, c \mid \vartheta) & =\left.\frac{\sqrt{t}}{\sigma(\vartheta)} F^{(0,1)}(x, y)\right|_{\begin{array}{l}
x=\frac{u}{\sigma(\vartheta) \sqrt{t}} \\
y=-\frac{(\vartheta-c) \sqrt{t}}{\sigma(\vartheta)}
\end{array}}
\end{aligned}
$$

7 Here and in what follows, the symbols $f^{(i, j)}\left(x_{1}, x_{2}\right)$ denote $i$-th and $j$-th partial derivatives of $f\left(x_{1}, x_{2}\right)$ with respect to the first and the second variables, respectively.

8 Note that Equation (1) rewrites as $\left.F(x, y)\right|_{x=\frac{u}{\sigma(\vartheta) \sqrt{t}}, y=-\frac{(\vartheta-c) \sqrt{t}}{\sigma(\vartheta)}}=\alpha$ where $x=\frac{u}{\sigma(\vartheta) \sqrt{t}}>0$ and $y=-\frac{(\vartheta-c) \sqrt{t}}{\sigma(\vartheta)}>0$. 
and

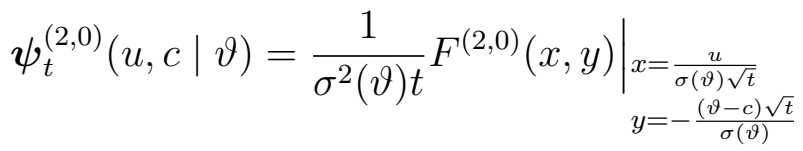

$$
\begin{aligned}
& \boldsymbol{\psi}_{t}^{(1,1)}(u, c \mid \vartheta)=\left.\frac{1}{\sigma^{2}(\vartheta)} F^{(1,1)}(x, y)\right|_{\begin{array}{l}
x=\frac{u}{\sigma(\vartheta) \sqrt{t}} \\
y=-\frac{(\vartheta-c) \sqrt{t}}{\sigma(\vartheta)}
\end{array}} \\
& \boldsymbol{\psi}_{t}^{(0,2)}(u, c \mid \vartheta)=\left.\frac{t}{\sigma^{2}(\vartheta)} F^{(0,2)}(x, y)\right|_{\substack{x=\frac{u}{\sigma(\vartheta) \sqrt{t}} \\
y=-\frac{(\vartheta-c \sqrt{t}}{\sigma(\vartheta)}}}
\end{aligned}
$$

Furthermore $^{9}$, for $x, y>0$

$$
\begin{aligned}
F^{(1,0)}(x, y) & =-2 \exp \{-2 x y\}\left(\varphi_{(0,1)}(-x+y)+y \Phi_{(0,1)}(-x+y)\right)<0 \\
F^{(0,1)}(x, y) & =-2 x \exp \{-2 x y\} \Phi_{(0,1)}(-x+y)<0 \\
F^{(2,0)}(x, y) & =4 y \exp \{-2 x y\}\left(\varphi_{(0,1)}(-x+y)+y \Phi_{(0,1)}(-x+y)\right) \\
& +2 x \exp \{-2 x y\} \varphi_{(0,1)}(-x+y) \\
F^{(1,1)}(x, y) & =-2 \exp \{-2 x y\} \Phi_{(0,1)}(-x+y)+4 x y \exp \{-2 x y\} \Phi_{(0,1)}(-x+y) \\
& +2 x \exp \{-2 x y\} \varphi_{(0,1)}(-x+y) \\
F^{(0,2)}(x, y) & =2 x \exp \{-2 x y\}\left(2 x \Phi_{(0,1)}(-x+y)-\varphi_{(0,1)}(-x+y)\right)
\end{aligned}
$$

Direct algebraic manipulations yield

$$
\begin{aligned}
& F^{(0,2)}(x, y)\left(F^{(1,0)}(x, y)\right)^{2}-2 F^{(1,1)}(x, y)\left(F^{(1,0)}(x, y) F^{(0,1)}(x, y)\right) \\
& +F^{(2,0)}(x, y)\left(F^{(0,1)}(x, y)\right)^{2}=8 x\left(\exp \{-2 x y\} \varphi_{(0,1)}(-x+y)\right)^{3} D(x, y)
\end{aligned}
$$

where

$$
D(x, y)=2 y \mathcal{M}^{3}(x-y)+\left(2+x^{2}-y^{2}\right) \mathcal{M}^{2}(x-y)-2 y \mathcal{M}(x-y)-1
$$

and (see Section 4.2)

$$
\mathcal{M}(x-y)=\frac{1-\Phi_{(0,1)}(x-y)}{\varphi_{(0,1)}(x-y)}=\frac{\Phi_{(0,1)}(-x+y)}{\varphi_{(0,1)}(-x+y)}
$$

is Mill's ratio. Note that

$$
D(x, y)=D_{1}(x, y)+D_{2}(x, y)
$$

where

$$
D_{1}(x, y)=2 x \mathcal{M}^{3}(x-y)+2 x(x-y) \mathcal{M}^{2}(x-y)-2 x \mathcal{M}(x-y)
$$

and

$$
D_{2}(x, y)=-2(x-y) \mathcal{M}^{3}(x-y)+\left(2-(x-y)^{2}\right) \mathcal{M}^{2}(x-y)+2(x-y) \mathcal{M}(x-y)-1
$$

9 To observe that $F^{(1,0)}(x, y)<0$ for $x, y>0$, note that $F^{(1,0)}(x, y)=-2 \exp \{-2 x y\} \varphi_{(0,1)}(-x+y)(1+y \mathcal{M}(x-y))$. For $w=x-y \in \mathrm{R}$, we have $1+y \mathcal{M}(x-y)=1+(x-w) \mathcal{M}(w)=(1-w \mathcal{M}(w))+x \mathcal{M}(w)$, which is positive since both summands are positive. 
Prove that $D(x, y)>0$ for $x, y>0$. Bearing in mind that $1-w \mathcal{M}(w)<\mathcal{M}^{2}(w)$ and $\mathcal{M}(w)>0$ for any finite $w$ (see inequalities (14)), it follows from

$$
\begin{aligned}
D_{1}(x, y) & =\left.\left(2 x \mathcal{M}^{3}(w)+2 x w \mathcal{M}^{2}(w)-2 x \mathcal{M}(w)\right)\right|_{w=x-y \in \mathrm{R}} \\
& =\left.2 x\left(\mathcal{M}^{2}(w)+w \mathcal{M}(w)-1\right) \mathcal{M}(w)\right|_{w=x-y \in \mathrm{R}}>0
\end{aligned}
$$

true for $x>0$, and from

$$
\begin{aligned}
D_{2}(x, y) & =\left.\left(-2 w \mathcal{M}^{3}(w)+\left(2-w^{2}\right) \mathcal{M}^{2}(w)+2 w \mathcal{M}(w)-1\right)\right|_{w=x-y \in \mathrm{R}} \\
& =\left.\left((1-w \mathcal{M}(w))\left(2 \mathcal{M}^{2}(w)-(1-[w \mathcal{M}(w)])\right)\right)\right|_{w=x-y \in \mathrm{R}} \\
& >\left.((1-w \mathcal{M}(w))(2(1-w \mathcal{M}(w))-(1-w \mathcal{M}(w))))\right|_{w=x-y \in \mathrm{R}} \\
& =\left.\left((1-w \mathcal{M}(w))^{2}\right)\right|_{w=x-y \in \mathrm{R}}>0
\end{aligned}
$$

We finally have for all $c>\vartheta$ and $u>0$

$$
\frac{\partial^{2}}{\partial c^{2}} u_{\alpha, t}(c \mid \vartheta)=-\left.\frac{t^{3 / 2}}{\sigma(\vartheta)} \frac{D(x, y)}{\left(F^{(1,0)}(x, y)\right)^{3}}\right|_{\substack{x=\frac{u}{\sigma(\vartheta) \sqrt{t}} \\ y=-\frac{(\vartheta-c) \sqrt{t}}{\sigma(\vartheta)}}}>0
$$

and the proof is complete.

Proof of Theorem 4 Bearing in mind the convexity ${ }^{10}$ of $u_{\alpha, t}(c \mid \vartheta)$ established in Theorem 5, apply Theorem 7 with $A=u_{\alpha, t}(\vartheta \mid \vartheta), B=\left(-\sigma^{2}(\vartheta) \ln \alpha\right) / 2, C=\vartheta$. We have the straight line

$$
l(c)=-\left(A^{2} / 4 B\right)(c-C)+A=\frac{t \kappa_{\alpha / 2}^{2}}{2 \ln \alpha}(c-\vartheta)+\sigma(\vartheta) \sqrt{t} \kappa_{\alpha / 2}
$$

tangent to the hyperbola

$$
h(c)=B /(c-C)=-\frac{\sigma^{2}(\vartheta) \ln \alpha}{2}(c-\vartheta)^{-1}
$$

This hyperbola is (see Theorem 3) the upper bound for $u_{\alpha, t}(c \mid \vartheta)$, as $c>\vartheta$.

Abscissa and ordinate of the point of tangency are $\vartheta-\sigma(\vartheta) \ln \alpha /\left(\sqrt{t} \kappa_{\alpha / 2}\right)$ and $\sigma(\vartheta) \sqrt{t} \kappa_{\alpha / 2} / 2$. Taking the tangent line as the bound for $\vartheta<c \leqslant \vartheta-\sigma(\vartheta) \ln \alpha /\left(\sqrt{t} \kappa_{\alpha / 2}\right)$ and the hyperbola as the bound for $c>\vartheta-\sigma(\vartheta) \ln \alpha /\left(\sqrt{t} \kappa_{\alpha / 2}\right)$, we complete the proof.

Proof of Corollary 3 It follows straightforwardly from Theorem 4 and from the fact that $c_{\alpha, t}(u \mid \vartheta)=$ $u_{\alpha, t}^{-1}(u \mid \vartheta)$ for any $u>0$.

10 The graph of a convex function $u(c), c \in\left[c_{1}, c_{2}\right]$, lies below the straight line connecting the points with abscissa $c_{1}$ and ordinate $w_{1}$ and with abscissa $c_{2}$ and ordinate $w_{2}$, where $w_{1} \geqslant u\left(c_{1}\right)$ and $w_{2} \geqslant u\left(c_{2}\right)$. 


\section{Auxiliary Results}

\subsection{Mill's Ratio}

For finite $x$, Mill's ratio is defined as

$$
\mathcal{M}(x)=\frac{1-\Phi_{\{0,1\}}(x)}{\varphi_{\{0,1\}}(x)}=e^{x^{2} / 2} \int_{x}^{\infty} e^{-t^{2} / 2} d t
$$

Evidently, $\mathcal{M}(x)>0$ for all finite $x$. The most well-known results about Mill's ratio are $\mathcal{M}^{\prime}(x)=$ $x \mathcal{M}(x)-1<0$ and $\mathcal{M}^{\prime \prime}(x)=\mathcal{M}(x)\left(1+x^{2}\right)-x>0$, so $\mathcal{M}(x)$ is convex and decreasing from $\infty$ to 0 , as $x$ increases from $-\infty$ to $+\infty$.

The inequalities ${ }^{11}$

$$
0<1-x \mathcal{M}(x)<\mathcal{M}^{2}(x)
$$

were proved in [6]. For this purpose, it has been observed that the function $e^{-t^{2} / 2} / \int_{x}^{\infty} e^{-t^{2} / 2} d t$ is a p.d.f. over the range $x \leqslant t<\infty$, and its variance is $1-\nu(x)(\nu(x)-x)$, where $\nu(x)=e^{-x^{2} / 2} / \int_{x}^{\infty} e^{-t^{2} / 2} d t$ is the reciprocal of Mill's ratio. Since this must be positive for finite $x$, the inequality $\nu(x)(\nu(x)-x)<1$ follows. It is the upper limit in (14).

\subsection{Differentiation of Implicit Functions}

The following implicit function theorem is well known in analysis (see e.g., Chapter I, $\S 5.2$ and $\S 5.3$ in [7]).

Theorem 6 Let $F$ be a function that possesses partial derivatives up to second order continuous in some neighborhood of some solution, $\left(x_{0}, y_{0}\right)$, of the equation $F(x, y)=0$. If $F_{2}\left(x_{0}, y_{0}\right) \neq 0$, there are an $\epsilon>0$ and a unique continuously differentiable function $\phi$ such that $\phi\left(x_{0}\right)=y_{0}$ and $F(x, \phi(x))=0$ for $\left|x-x_{0}\right|<0$. Moreover, when $\left|x-x_{0}\right|<0$, we have

$$
\phi^{\prime}(x)=-F^{(1,0)}(x, \phi(x))\left(F^{(0,1)}(x, \phi(x))\right)^{-1}
$$

and

$$
\begin{aligned}
& \phi^{\prime \prime}(x)=-\left(F^{(2,0)}(x, \phi(x))\left(F^{(0,1)}(x, \phi(x))\right)^{2}\right. \\
& -2 F^{(1,1)}(x, \phi(x)) F^{(1,0)}(x, \phi(x)) F^{(0,1)}(x, \phi(x)) \\
& \left.\quad+F^{(0,2)}(x, \phi(x))\left(F^{(1,0)}(x, \phi(x))\right)^{2}\right)\left(\left(F^{(0,1)}(x, \phi(x))\right)^{3}\right)^{-1}
\end{aligned}
$$

11 The lower limit in (14) is easy. Since $\frac{d}{d x}(x \mathcal{M}(x))=\mathcal{M}^{\prime \prime}(x)>0$ for finite $x$, the function $x \mathcal{M}(x)$ is increasing from $-\infty$ to 1 , as $x$ increases from $-\infty$ to $+\infty$. 


\subsection{Straight Line and Hyperbola}

The following theorem is straightforward.

Theorem 7 For $A, B, C>0$, the straight line $l(x)=-\left(A^{2} / 4 B\right)(x-C)+A$ with a negative slope passing through the point with abscissa $C$ and ordinate $A$, is tangent to the hyperbola $h(x)=B /(x-C)$ at the point with abscissa $2 B / A+C$ and ordinate $A / 2$.

\section{Acknowledgments}

This work was supported by RFBR (grant No. 11-06-00057-a).

\section{Conflicts of Interest}

The authors declare no conflicts of interest.

\section{References}

1. Borodin, A.N.; Salminen, P. Handbook of Brownian Motion. Facts and Formulae; Birkhäuser: Basel, Switzerland, 1996.

2. Malinovskii, V.K. Zone-adaptive control strategy for a multiperiodic model of risk. Ann. Actuar. Sci. 2007, 2, 391-409.

3. Malinovskii, V.K. Scenario analysis for a multi-period diffusion model of risk. ASTIN Bull. 2009, 39, 649-676.

4. Malinovskii, V.K. Level premium rates as a function of initial capital. Insur.: Math. Econ. 2013, $52,370-380$.

5. Malinovskii, V.K. Improved asymptotic upper bounds on ruin capital in Lundberg model of risk. Insur:: Math. Econ. 2014, 55, 301-309.

6. Sampford, M.R. Some inequalities on Mill's ratio and related functions. Ann. Math. Stat. 1953, 24, 130-132.

7. Widder, D.V. Advanced Calculus; Prentice-Hall: New York, NY, USA, 1947.

(c) 2014 by the author; licensee MDPI, Basel, Switzerland. This article is an open access article distributed under the terms and conditions of the Creative Commons Attribution license (http://creativecommons.org/licenses/by/3.0/). 